\title{
The Feeding Rate Optimization Based on Differential Evolution Algorithm
}

\author{
Hua Meng ${ }^{1, a}$, Liting $\operatorname{Han}^{2, b^{*}}$ and Xiaohong Zheng ${ }^{3, c}$ \\ ${ }^{123}$ School of Electrical Engineering Hebei University of Science and Technology Shijiazhuang, \\ Hebei Province, 050000, China \\ amenghua@162.com, b18732182156@163.com, c15614120310@163.com \\ ${ }^{*}$ The corresponding author
}

\begin{abstract}
Keywords: Fermentation process; Feeding; Differential evolution algorithm; Process optimization
\end{abstract}

\begin{abstract}
Bacteria concentration is one of the important parameters throughout the fermentation process, and in the whole process of fermentation it is an important basis for judging the feeding. Bacteria concentration in the end is the important factor that affects product concentration, so the appropriate bacteria concentration is a top priority in the fermentation process. Pichia expression of recombinant protein fermentation process acts as the research background, and the differential evolution (DE) algorithm is applied to optimize the feeding. The propose is to get more bacteria concentration by optimizing glycerol and ammonia feeding rate in bacteria growth stage. The simulation results indicate that the method is effective in solving the optimization problem of feeding rate, and that the feasibility of the optimization algorithm in the fermentation process is verified.
\end{abstract}

\section{Introduction}

Optimization problem which we discuss and research is to find the optimal solution in many scenarios [1]. In the past few years, the interaction of computer science and optimization research have played an extremely important role in the development of intelligent optimization technology. Therefore, evolutionary algorithm represents the common areas of optimization and computer science. The structure of the algorithm is inspired from the mechanism of human evolution, and DE is the most closed to evolutionary algorithm. Differential evolution algorithm has good performance to research the solution [2]. The differential evolution algorithm was applied to the fermentation process in this article, and the mathematical model of the cell concentration and the adding nutrients was established based on BP neural network.

\section{Fermentation Process Modeling Based on Neural Network}

There are four phases in Pichia expression of recombinant protein fermentation process such as hysteresis phase, bacteria growth phase, inducible expression phase and decline phase. The total fermentation cycle is 190 hours in actual production process. And the bacteria growth phase is 24 hours. The appropriate bacteria concentration is obtained before inducible expression phase. In the bacteria growth stage, in order to obtain more cell growth, carbon and nitrogen has been decreased rapidly. Ammonia as nitrogen adjusts $\mathrm{pH}$, and glycerol as carbon is used as nutrient which is the need of bacteria growth.

Neural network has highly nonlinear mapping ability [3]. The model can be built only rely on the input and output. It applies to the complex nonlinear process as the same as fermentation process [4]. The excellent model which is built need to get the sample, dispose data, design the network structure, train the network model, and verify the network model.100 batches of data is used in this article. It is divided in two parts. One is training data, and the other is testing data. 80 batches of data is used as the training sample, and 20 batches of data is used as the testing sample. The model of bacteria concentration is built that based on BP neural network. The BP neural network model 
which is simulated by MATLAB can accurately reflect the fermentation process. As the Fig. 1 shown, the bacteria concentration model is based on BP neural network.

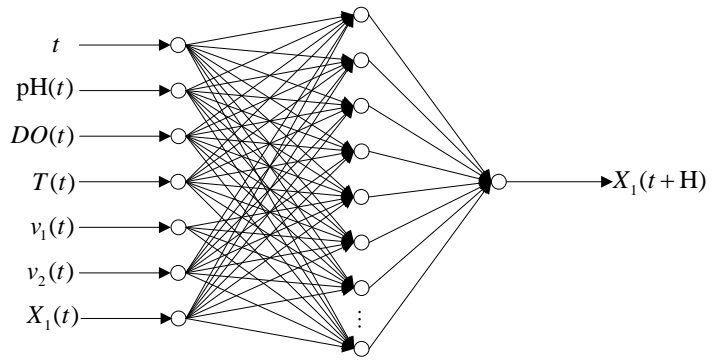

Figure 1. Bacteria concentration model based on BP neural network

The model structure is Type 7-9-1. It means that 7 inputs variable afferent the input layer of neural network, and the number of hidden layer is 9 , and one output variable outputs in output layer. Training sample is used to train the designed network [5]. Threshold and weight are revised continually until the error precision of network output has been satisfied the error precision of expectation.

\section{The Feeding Optimization Control of Fermentation Process Based on Differential Evolution}

Differential Evolution. The basic operations of differential evolution include mutation, crossover and selection [6]. A differential vector that results from subtracting two individual vectors. The two individual vectors are selected by randomly. After the differential vector is multiplied by the weight, the result added to the third individual vector which is selected randomly. Then the mutational vector is produced. This operation is called mutation. The parameters of mutational vector and objective vector are mixed. It will produce experimental vector. This operation is called crossover. If the fitness of experimental vector is better than the fitness of the objective vector, the experimental vector replaces the objective vector. So it form the next generation. This operation is called selection. In the process of the evolution of every generation, each individual vector is regarded as the target vector one time. Initial population is randomly generated in the search space. And initial population are required to cover the whole search space. The initial population are generated by uniformly distributed function.

The Description of Optimization Problem. In the bacteria growth stage, the bacteria concentration directly influences the fermentation product concentrate [7]. Based on the model established by neural network, the DE algorithm is adopted to respectively optimize ammonia feeding rate and glycerol feeding rate of the bacteria growth stage. The objective function is followed:

$$
J=\max _{v_{1}(t), v_{2}(t)} X_{1}(t+H), 0 \leq t \leq t_{i}
$$

And the constraints are displayed as followed:

$$
\begin{aligned}
& X_{1}(t+H)=f\left(t, T(t), p \mathrm{H}(t), D O(t), X(t), v_{1}(t), v_{2}(t)\right) \\
& v_{1}(t)_{\min } \leq v_{1}(t) \leq v_{1}(t)_{\max } \\
& v_{2}(t)_{\min } \leq v_{2}(t) \leq v_{2}(t)_{\max } \\
& T(t)=30^{\circ} \mathrm{C} \\
& p H(t)=5.0
\end{aligned}
$$




$$
D O(t)=75 \%
$$

Where $t_{i}$ is the end moment in the bacteria growth stage, $X(t)$ is bacteria concentration, $t$ is fermentation time, $T(t), p H(t), D O(t), v_{1}(t), v_{2}(t)$ respectively denote temperature, dissolved oxygen, the ammonia feeding rate and the glycerol feeding rate at the $t$ moment, and $v_{1}(t)_{\min }, v_{2}(t)_{\max }$ respectively denote upper and lower of the ammonia feeding rate(In the actual fermentation process, where $\left.v_{1}(t)_{\min }=0, v_{1}(t)_{\max }=6(\mathrm{~L} / \mathrm{h})\right), v_{2}(t)_{\min }, v_{2}(t)_{\max }$ respectively denote upper and lower of the glycerol feeding rate(In the actual fermentation process, where $\left.v_{2}(t)_{\min }=0, v_{2}(t)_{\max }=1(\mathrm{~L} / \mathrm{h})\right), H$ is prediction cycle(in this article, $H=4 h$ ), and the temperature, dissolved oxygen and $\mathrm{pH}$ are constant. Based on the model by neural network the ammonia feeding rate and the glycerol feeding rate are calculated, and the optimal solution is solved. The optimization process in bacteria growth stage is followed. First of all, the neural model is established and the bacteria concentrate is the Objective function. It can simulate the fermentation process. Second, the optimal ammonia feeding rate and the optimal glycerol feeding rate are calculated by differential evolution algorithm. And the bacteria concentrate is obtained at $t$ instant. Finally, the feeding rate is optimized at $t+H, t+2 H$ instant, and bacteria concentrate is calculated. It is controlled by this method until the end of bacteria growth stage. The optimal feeding rate and bacteria concentrate can be concluded at each instant, and compared bacteria concentrate before and after optimization whether it satisfies the number of cells of expression of recombinant protein, whether it saves the total feeding, and whether it decreases the consumption of energy.

\section{Simulation Results}

Neural network is used to model in the bacteria growth stage, and DE is used to solve the optimal control. For a trained neural network, when a input has been given arbitrarily, a corresponding output will be received. Differential evolution algorithm can use this function to construct the fitness function. With rapidly reaching the bacteria concentration requirement for target, the optimization control mode which is proved to obtain the biggest bacteria concentrate at the end of bacteria growth stage in each period is established [8]. The feeding optimization of the twelfth to the sixteenth hour is used as an example, and the number of individuals in the population is 40 . The initial population distribution of the twelfth to the sixteenth hour is shown in Fig. 2.

In Fig. 2, the initial population distribution is uniform, and it is representative. Therefore, it can be used for the initial population distribution of DE. The optimization process of DE is described in Fig. 3.

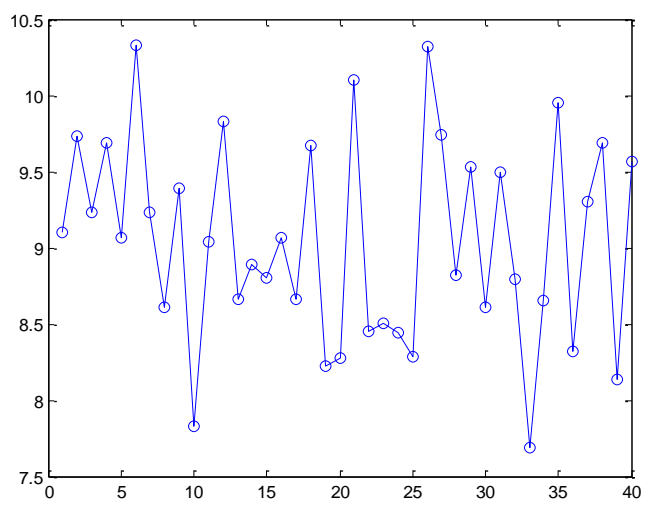

Figure 2. Initial population distribution [12h-16h]

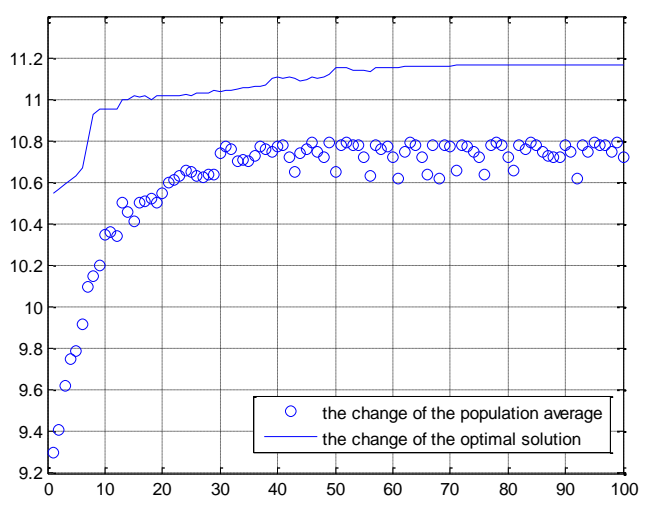

Figure 3. the evolution of the population[12h-16h]

As the picture shown, we discovered that the optimal individual is obtained in the 30 generations. The optimal gene decodes as followed.

$$
X=11.1240(\mathrm{~g} / \mathrm{mL}) \quad v_{1}=4.5094(L / h) \quad v_{2}=0.5040(\mathrm{~L} / \mathrm{h})
$$


It means that during the twelfth to the sixteenth hour when the glycerol feeding rate is $0.5040^{(L / h)}$, the ammonia feeding rate is $4.5094^{(L / h)}$, and this moment the bacteria concentration is $11.2640^{(\mathrm{g} / \mathrm{mL})}$.

And so on, the evolution is carried every four hours, and the feeding rate of each period of time and bacteria concentrate at that moment are acquired. In Table1, Table2, the before optimization and after optimization are compared. And we choose the maximum of all data without optimization for 'before optimization'.

As the Table1,Table2 shown, we can get that before optimization glycerol is consumed 41.2L and ammonia is consumed 7.2L, and after optimization glycerol is consumed $38.8 \mathrm{~L}$ and ammonia is consumed $4.2 \mathrm{~L}$. The bacteria concentrate after optimization is greater than that before optimization, and the consumption of glycerol and ammonia is respectively below the consumption of before optimization.

Table 1 The comparison of glycerol and ammonia feeding rate before and after optimization

\begin{tabular}{llcccc}
\hline \multirow{2}{*}{$\begin{array}{c}\text { fermentation } \\
\text { time } \\
{[\mathrm{h}]}\end{array}$} & \multicolumn{2}{c}{$\begin{array}{c}\text { glycerol feeding rate } \\
{[\mathrm{L} / \mathrm{h}]}\end{array}$} & \multicolumn{2}{c}{$\begin{array}{c}\text { ammonia feeding rate } \\
{[\mathrm{L} / \mathrm{h}]}\end{array}$} \\
\cline { 2 - 5 } & $\begin{array}{c}\text { before } \\
\text { optimization }\end{array}$ & $\begin{array}{c}\text { after } \\
\text { optimization }\end{array}$ & $\begin{array}{c}\text { Before } \\
\text { optimization }\end{array}$ & $\begin{array}{c}\text { After } \\
\text { optimization }\end{array}$ \\
\hline 0 & 0 & 0 & 0 & 0 \\
$0-4$ & 0 & 0 & 0 & 0 \\
$4-8$ & 0.2 & 0.2 & 0 & 0 \\
$8-12$ & 1.4 & 1.2 & 0 & 0 \\
$12-16$ & 4.6 & 4.5 & 0.6 & 0.5 \\
$16-20$ & 3.1 & 3.0 & 0.6 & 0.5 \\
$20-24$ & 1.0 & 0.8 & 0.6 & 0.05 \\
\hline
\end{tabular}

In order to make the optimized ammonia, glycerin loading trajectory more convenient and intuitive, we make the chart in Fig. 4 as the theoretical guidance of the actual fermentation industry.

Table 2 The comparison of bacteria concentration before and after optimization

\begin{tabular}{|c|c|c|}
\hline \multirow{2}{*}{$\begin{array}{c}\text { fermentation } \\
\text { time } \\
{[\mathrm{h}]}\end{array}$} & \multicolumn{2}{|c|}{ bacteria concentration $[\mathrm{g} / \mathrm{L}]$} \\
\hline & $\begin{array}{c}\text { before } \\
\text { optimization }\end{array}$ & $\begin{array}{c}\text { after } \\
\text { optimization }\end{array}$ \\
\hline 0 & 0 & 0 \\
\hline 4 & 0.401 & 0.561 \\
\hline 8 & 2.803 & 2.995 \\
\hline 12 & 5.934 & 6.326 \\
\hline 16 & 11.002 & 11.264 \\
\hline 20 & 14.013 & 15.747 \\
\hline 24 & 18.375 & 19.686 \\
\hline
\end{tabular}

As can be seen from Fig. 4, in the bacteria growth stage the basic process of Pichia expression of recombinant protein fermentation process is occurred in hysteresis phase. In the bacteria growth stage cells grow in geometric progression and the consumption of nutrient is increased, so the feeding is increased. 


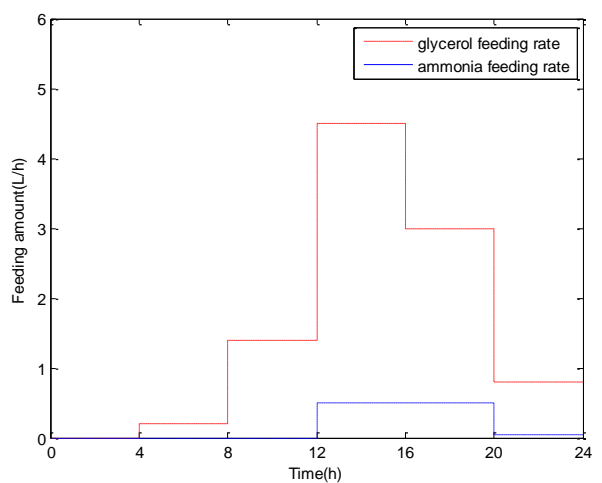

Figure 4. Optimal control curve of glycerol and ammonia feeding rate in the bacteria growth stage

\section{Summary}

Fermentation engineering technology promotes the industrialization of fermentation products [9]. The accurate control strategy can greatly improve the fermentation products [10]. The differential algorithm is a random heuristic search algorithm. It is not only simple but also stable. What's more, the ability of optimization in global is powerful. The differential evolution algorithm was applied to the fermentation process in this article. DE is applied the feeding rate to optimization. Compared the bacteria concentration before optimization to after optimization, it proved the effectiveness of differential evolution algorithm in optimization.

\section{References}

[1] Anne Richelle, Philippe Bogaerts. Off-line Optimization of Baker's Yeast Production Process. Chemical Engineering Science, 2014 (119):40-52.

[2] ZhongQuan Hu. The Optimization of Differential Evolution Algorithm and its Application Research:[Master Dissertation of Yanshan University].Hebei: Yanshan University,2013.

[3] B. MELIS, A. MUTLU. The Official Journal of the International Neural Network Society, 2013, 39: $18-26$

[4] JingWen Tian, MeiJuan Gao. Research and Application of BP neural network algorithm. Beijing: Beijing Institute of Technology press, 2008.

[5] C. SUN, M. G. JIANG. Construction and Application of GA-SVM Model for Personal Credit Scoring. Journal of Information and Computational Science, 2008, 5(2): 569-574

[6] DaPeng Niu, FuLi Wang, DaKuo He, Ming Xing Jia. Optimization of nosiheptide fermentation process based on the improved differential evolution algorithm for multi-objective optimization. Control Theory \& Applications. 2010(27) 504-508.

[7] Miguel Rocha, Rui Mendes, Orlando Rocha, Isabel Rocha, Eugénio C. Ferreira. Optimization of Fed-batch Fermentation Processes with Bio-inspired Algorithms. Expert Systems with Applications.2014 (41):2186-2195.

[8] P. KADLEC, B. GABRYS, S. STRANDT. Data-driven Soft Sensors in the Process Industry. Computers and Chemical Engineering, 2009, 33(4): 795-814

[9] QiLiang Fan. Research of the Subtrate Feeding Control Strategy in Fermentation Process: [Master Dissertation of Jiangnan University].Jiangnan: Jiangnan University, 2009.

[10] N. E. A. KHALID, N. M. NOOR, N, M, ARIFF, Fuzzy C-Means (FCM) for Optic Cup and Disc Segmentation with Morphological Operation. Procedia Computer Science, 2014(42):255-262 